\title{
Dissipativity and Feedback Dissipativity Properties of General Nonlinear Discrete-Time Systems*
}

\author{
Eva María Navarro-López ${ }^{1, \dagger}$, Hebertt Sira-Ramírez ${ }^{2, \neq}$ and Enric Fossas-Colet ${ }^{1}$ \\ 'Institut d’Organització i Control, Universitat Politècnica de Catalunya. Avda. Djagonal 647, 11 planta, 08028 Barcelona, Spain; \\ ${ }^{2}$ CINVESTAV-IPN. Departamento de Ingenieria Eléctrica, Sección de Mecatrónica, Colonia San Pedro Zacatenco, A. P. 14-740, \\ 07300 México, D.F., México
}

The dissipativity of single-input single-output discretetime systems which are nonlinear in the states and the control input is examined. Necessary conditions for the characterization of what is regarded as $(\boldsymbol{V}, \mathrm{s})$-dissipative systems are given; in the special case of Quadratic Storage Supply lossless systems, necessary and sufficient conditions are proposed. The results obtained are compared with the ones already existing in the literature for losslessness, passivity and dissipativity cases. Sufficient conditions under which a class of non-affine discrete-time control systems can be rendered dissipative are derived. A procedure for dealing with feedback dissipativity and passivation problems is proposed, in addition to the extension of the Energy Shaping plus Damping Injection controller design method for the discrete-time case. A discrete-time model for the $D C-10-D C$ buck converter is used as an example to illustrate the control scheme proposed.

Keywords: Discrete-time systems; Dissipativity; Dissipativity-based control; Feedback dissipativity; Nonlinear systems

'Partially supported by CICYT under Grant. n. DPL2000-1509$\mathrm{CO}-02$

${ }^{\dagger}$ Under fellowship from Ministerio de Educación y Cultura and Consejo Superior de Investigaciones Cientificas (Spain). E-mail: enavarro99(a)prodigy.net.mx

tPartially supported by Consejo Nacionat de Ciencia y Tecnología de México (CONACYT) under research contract n. 32681-A. E-mail: hsirata:mail.cinvestav,mx

Correspondence and offprint requests to: E. Fossas-Colet, Institut d'Organització i control. Universitat Politècnica de Catalunya, Avda. Diagonal 647, 11 planta, 08028 Barcelona. Spain. Tel.: 34-934017779; Fax: 34-93-4016605. E-mail: fossas $(a$ ioc.upc.es

\section{Introduction}

The concepts of passivity and dissipativity have received considerable attention in the last decades. Dissipative systems present highly desirable stability properties, which may simplify analysis and controller design. A dissipative system is a system which cannot store all the supplied energy, i.e., it dissipates energy. A dissipative system is characterized by the existence of a storage function and a supply function.

The first ideas of passivity emerged in the field of circuit theory, from the phenomenon of dissipation of energy across resistors see, e.g., Zames [34]. The formal definitions were introduced by Willems [32] in the early $1970 \mathrm{~s}$ in terms of the storage and supply rate functions. A different research line was initiated by $\mathrm{Wu}$ and Desoer [33], cast in terms of the system input-output properties from a general operator theory viewpoint. A significant advance was made by Popov [24], who established passivity as an important feedback property using the concepts of hyperstability and absolute stability. The extension of dissipativity results to nonlinear systems which are affine in the control input was given by $\mathrm{Hill}$ and Moylan $[6,7,18]$.

The most important formalization of the characteristics of a passive system is the KalmanYakubovich-Popov (KYP) property or the KYP lemma. Although originally the KYP lemma established the connection between passivity conditions (i.e., a set of properties that any passive system fulfils)

Received 4 December 2000: Accepted in revised form 17 Apri/ 20012. Recommended by A. Astolfi and I. D. Landau. 
and positive real transfer functions (see e.g., Khali] [10]), the denomination of KYP property has been adopted to address the set of necessary and sufficient conditions that a passive system satisfies, irrespectively of its linear or nonlinear character. In the sequel, we use the KYP denomination in this sense.

As dissipative and passive systems have very interesting properties, a natural motivation is to transform a system which is not dissipative or passive into a dissipative or passive one. The action of rendering a system dissipative (respectively, passive) by means of a static state feedback is known as feedback dissipativity (respectively, feedback passivity or passivation). Systems which can be rendered dissipative (respectively, passive) are regarded as feedback dissipative (respectively, feedback passive) systems. The problem of feedback passivity was first posed and solved by Kokotović and Sussmann [1 I] introducing the concept of feedback positive systems. The study of feedback passive systems is given by the work of Byrnes et al. [1], where necessary and sufficient conditions for passivation of nonlinear affine-in-control continuous-time systems are established in terms of the system's relative degree and zero dynamics. These results were later extended by Santosuosso [25] to systems having an affine feedthrough term between the input and the output. An alternative approach to the one presented by Byrnes for solving the passivation problem is presented by Sira-Ramirez [30]. Interesting surveys of passivity and passivation properties and their implications in dynamical systems and in connection to the stabilization problem are given in Sepulchre et al. [28], van der Schaft [26], Ortega et al. [22] and Fradkov and Pogromsky [4].

All the aforementioned passivity-related results are cast in the context of affine-in-control continuoustime systems. The non-affine continuous-time case is examined in Lin $[14,17]$, where necessary conditions for a non-affine nonlinear continuous system to be passive are given. Furthermore, passivity properties are used in order to achieve asymptotic stabilization. The use of passivity for the stabilization of non-affine systems is also addressed in Shiriaev [29] by means of the $V$-detectability concept, a generalization of zerostate detectability [1]. Moreover, the problem of passivation is solved by means of the speed-gradient algorithm [4]. Another recent approach which motivates this work is the one given by Sira-Ramirez and Navarro-López [31].

Concerning the extension of dissipativity and passivity for the nonlinear discrete-time case, several approaches can be distinguished. The initial results were given by $\mathrm{Wu}$ and Desoer [33] in terms of input-output properties, connecting passivity with feedback stabilization. Most of the available results referring to passivity in the discrete-time domain are given by Byrnes and $\operatorname{Lin}[2,3,15,16]$ and can be considered as the extension of the philosophy underlying Byrnes $2 t$ al. [1]. They give necessary and sufficient conditions for an affine-in-control discrete-time nonlinear system to be lossless and feedback lossless via a static state feedback. A characterization of affine discrete-time passive systems is also presented [2]. Necessary conditions for a system to be passive are proposed in a different line in Lin and Byrnes [5]. which are generalized for the non-affine case [14]. Connections between passivity and stabilization are given in Lin and Byrnes $[3,13,15,16]$. Sengör and Göknar's [5,27] provide a KYP Lemma for dissipativity and losslessness for discrete-time affinein-control nonlinear systems. They use the definition of gradient-like functions and adapt the necessary and sufficient conditions for dissipativity proposed in Hill and Moylan [6]. Another approach to passivity in the discrete-time case is represented by the work of Monaco and Normand-Cyrot [19]. They obtain the KYP conditions for general non-affine discrete nonlinear systems which can be expanded by exponential Lie series.

For the discrete-time case many dissipativityrelated problems remain unsolved. The main problem being the establishment of conditions for a discretetime system to be rendered dissipative or passive via state feedback. The only results referring to feedback dissipativity in nonlinear discrete-time systems are the ones related to making an affine-in-control system lossless by means of static state feedback using the properties of the relative degree and zero dynamics of the system [3].

The purpose of this paper is twofold. First, we will give some properties for dissipative and passive nonlinear discrete-time systems and an implicit solution for feedback dissipativity and passivation problems. Second, these results will be used to tackle the stabilization problem. The stabilization procedure will be an extension of the Energy Shaping plus Damping Injection (ESDI) methodology existing for the continuous-time nonlinear case (see $[23,30]$ ).

The paper is organized as follows. Section 2 deals with several topics. First, the basic definitions about dissipative systems and feedback dissipative systems for the discrete-time case are revisited. Second, necessary conditions for the characterization of a class of dissipative systems, in addition to necessary and sufficient conditions for the characterization of Quadratic Storage Supply lossless systems are derived for the non-affine case different to the ones presented in the literature. Third, sufficient conditions under 
which a class of non-affine discrete-time control systems can be rendered dissipative are posed. Finally, stability properties of a class of dissipative and feedback dissipative systems are examined. Section 3 presents the extension of the ESDI design method to nonlinear discrete-time systems. Section 4 presents the application of the ESDI to a discrete-time model for the DC-to-DC buck converter. Conclusions and suggestions for further research are presented in the last section.

\section{Dissipativity and Passivity of General Discrete-Time Nonlinear Systems}

\subsection{Generalities}

Let nonlinear single-input single-output discrete-time systems of the form

$$
\begin{aligned}
& x(k+1)=f(x(k), u(k)), \\
& x \in \mathscr{x} \subset \mathbb{R}^{\prime \prime}, \in \mathscr{U} \subset \mathscr{R}, \\
& y(k)=h(x(k), u(k)), \quad y \in \mathscr{Y} \subset \mathscr{x},
\end{aligned}
$$

where $f: \mathscr{X} \times \mathscr{U} \rightarrow \mathscr{X}$ and $h: \not \mathscr{X} \times \mathscr{U} \rightarrow \mathscr{y}$ are smooth maps. $k \in \mathscr{Z}_{+}:=\{0,1,2, \ldots\}$. All considerations will be restricted to an open set of $\mathscr{P} \times \mathscr{U}$ containing $(\bar{x}, \bar{u})$, having $\bar{x}$ as an isolated fixed point of $f(x, \bar{u})$, with $\bar{u}$ a constant, i.e., $f(\bar{x}, \bar{u})=\bar{x}$. We consider a positive definite $\mathscr{C}^{2}$ function $V: \mathscr{T} \rightarrow \mathscr{P}$ associated with the system (1)-(2) and addressed as the storage function. Function $V$ is considered to have a strict local minimum in $\bar{x}$, i.e., there exists an open neighbourhood $\bar{x}$ of $\bar{x}$ such that $V(\bar{x})<V(x), \forall x \in \bar{x}$. A second $\mathscr{C}^{2}$ function is also considered, called the supply function, denoted by $s(y, u)$, with $s: \mathscr{Y} \times \mathscr{U} \rightarrow \mathscr{R}$.

Definition 2.1. The supply function $s(y, u)$ is said to satisfy the zero-input-output (ZIO) property if

$$
\begin{array}{ll}
s(0, u)=0, & \forall u \in \mathscr{U}, \\
s(y, 0)=0, & \forall y \in \mathscr{Y} .
\end{array}
$$

Definition 2.2. A $\mathscr{C}$ function $\phi: \mathscr{X} \times \mathscr{U} \rightarrow \not{R}$, such that $\phi(\cdot, u)$ is positive (respectively, strictly positive) for each $u \in \mathscr{U}$, with $(\bar{x}, \bar{u})$ a strict local minimum of $\phi$ is regarded as a dissipation rate (resp., strict dissipation rate) function in the sense proposed in Hill and Moylan [7].

The dissipativity definition in the discrete-time nonlinear setting given in Byrnes and Lin [3] will be rewritten in the following way.

Definition 2.3. The system (1)-(2) with storage function $V(x)$ and supply function $s(y, u)$ is said to be
$(V, s)$-dissipative (resp., strictly $(V, s)$-dissipative) if there exists a dissipation rate (resp., strict dissipation rate) function $\phi$ such that

$$
\begin{gathered}
V(f(x, u))-V(x)=s(h(x, u), u)-\phi(x, u), \\
\forall(x, u) \in X \times \mathscr{U}
\end{gathered}
$$

Definition 2.4. The system (1)-(2) is said to be $V$-passive if it is $(V, s)$-dissipative with respect to the supply rate $s(y, u)=y u$. The system is said to be $(V, s)$-lossless if $\phi(x, u)=0, \forall(x, u) \in \mathscr{X} \times \mathscr{U}$.

$(V, s)$-dissipativity and $V$-passivity have immediate consequences referring to the stability of the system when no control is applied and the stability of the zero dynamics, as it will be illustrated in Theorem 2.6. Lyapunov's Stability Theorem for discrete-time systems is presented, since it will be used in the sequel.

Theorem 2.5. (LaSalle [12] Lyapunov's Stability Theorem). Consider a discrete-time system of the form $x(k+1)=f(x(k))$. Let $\bar{x}$ be a fixed point of the system. The system dynamics will be restricted to a neighbourhood $S$ of $\bar{x} \in \mathscr{R}^{\prime \prime}, f: S \rightarrow S$. Suppose that there exists a continuous, positive definite function $V: S \rightarrow \mathscr{R}^{+}$, with $V(\bar{x})=0$. If $V(x(k+1))-$ $V(x(k)) \leq 0, \forall x \in S$, then $\bar{x}$ is a stable equilibrium (if $V(x(k+1))-V(x(k))<0$, then $\bar{x}$ is asymptotically stable).

Theorem 2.6. For $(V, s)$-dissipative systems with positive definite storage functions and supply functions satisfying the ZIO property, the fixed point, $\bar{x}$, of the zero-input dynamics $x(k+1)=f(x(k), 0)$ is Lyapunov stable (resp., asymptotically stable if the system is strictly $(V, s)$-dissipative). Similarly, if the output $y$ of this class of dissipative systems is held to be zero in an indefinite fashion by means of an appropriate control input, then the zero dynamics is Lyapunov stable (resp., asymptotically stable if the system is strictly ( $V, s)$-dissipative).

Proof. These statements can be proved restricting (4) for $u=0$ in the first case and for $y=0$ in the second one, and considering conditions (3) in addition to Lyapunov's Stability Theorem 2.5 .

\subsection{Towards the Characterization of General Discrete-Time Dissipative Systems}

The properties that a system has to meet in order to be dissipative or passive are usually known as the KYP conditions. The KYP conditions were originally established in the discrete-time domain by $\mathrm{Hitz}$ and Anderson [8] for the linear case as the Positive Discrete 
Real Lemma. In the literature, the KYP conditions have not been established in a general way for dissipative or passive nonlinear discrete-time systems of the form (1)-(2). There exist necessary and sufficient conditions for characterizing passive nonlinear discrete-time systems of the affine form $x(k+1)=$ $f(x(k))+g(x(k)) u(k), y(k)=h(x(k))+J(x(k)) u(k)$, presumed the stored energy function $V$ satisfies $V(f(x)+$ $g(x) u)$ is quadratic in $u$ [2]. Necessary conditions for systems of the form (1)-(2) to be passive are stated in Lin [14]. Moreover, the necessary and sufficient conditions for dissipativity in the discrete-time setting appearing in Göknar and Sengör [5] are proposed for affine-in-control system structures as well.

We remark that, in the literature, the KYP denomination is usually used for the set of properties for dissipative or passive systems characterization, even if they are only necessary conditions. In this sense, we propose the following conditions, which are fulfilled by any nonlinear discrete-time dissipative system of the form (1)-(2). The corresponding result for passive systems is obtained taking $s=y u$.

Proposition 2.7. Let a discrete-time system of the form (1)-(2) be $(V, s)$-dissipative, then

$$
\begin{aligned}
V(f(x, 0))-V(x) & \leq s(h(x, 0), 0) \\
\frac{\partial}{\partial u} V(f(x, u))= & \left.\frac{\partial}{\partial u_{2}} s\left(h\left(x, u_{1}\right), u_{2}\right)\right|_{\substack{u_{1}=u \\
u_{2}=u}} \\
& +\frac{\partial}{\partial y} s(y, u) \frac{\partial}{\partial u} h(x, u) \\
& -\frac{\partial}{\partial u} \phi(x, u),
\end{aligned}
$$

with $\phi$ a dissipation rate function.

Proof. Inequality (5) follows from (4), taking $u=0$. Equality (6) is obtained by taking partial derivatives with respect to $u$ in Eq. (4).

Remark 2.8. The necessary conditions for passive systems of the form (1)-(2) given in Lin [14] are different from the ones given in (5) and (6). In Lin's work, the passivity inequality is used and no dissipation rate function $\phi$ is introduced.

Conditions (5) and (6) may be rewritten for the losslessness case in the following way.

Proposition 2.9. Let a discrete-time system of the form (1) $-(2)$ be $(V, s)$-lossless, then

$$
V(f(x, 0))-V(x)=s(h(x, 0), 0)
$$

$$
\begin{aligned}
\frac{\partial}{\partial u} V(f(x, u))= & \left.\frac{\partial}{\partial u_{2}} s\left(h\left(x, u_{1}\right), u_{2}\right)\right|_{\substack{u_{1}=u \\
u_{2}=u}} \\
& +\frac{\partial}{\partial y} s(y, u) \frac{\partial}{\partial u} h(x, u)
\end{aligned}
$$

If functions $V(f(x, u))$ and $s(h(x, u), u)$ are quadratic in $u$, then equalities (7)-(8) yieid necessary and suffcient as Theorem 2.11 shows. Therefore, our characterization is restricted to a class of lossless systems.

Definition 2.10. A system of the form (1) (2) is said to be QSS (Quadratic Storage Supply)-lossless if it is $(V, s)$-lossless with a storage function $V$ and a supply function $s$ such that $V(f(x, u))$ and $s(h(x, u), u)$ are quadratic in $u$.

Theorem 2.11. Let $V$ be a storage function and $s$ be a supply function such that $V(f(x, u))$ and $s(h(x, u), u)$ are quadratic in $u$. Then, a system of the form (1) - (2) is QSS-lossless with $V$ and $s$, if and only if

$$
\begin{aligned}
& V(f(x, 0))-V(x)=s(h(x, 0), 0) \\
& \left.\left.\frac{\partial V(z)}{\partial z}\right|_{z=f(x, 0)} \frac{\partial f(x, u)}{\partial u}\right|_{u=0}=\left.\frac{\partial}{\partial u} s(h(x, u), u)\right|_{u=0} \\
& \left.\left.\left.\left(\frac{\partial f(x, u)}{\partial u}\right)\right|_{u=0} ^{\mathrm{T}} \frac{\partial^{2} V(z)}{\partial z^{2}}\right|_{z=f(x, 0)} \frac{\partial f(x, u)}{\partial u}\right|_{u=0} \\
& +\left.\left.\frac{\partial V(z)}{\partial z}\right|_{z=f(x, 0)} \frac{\partial^{2} f(x, u)}{\partial u^{2}}\right|_{u=0} \\
& =\left.\frac{\partial^{2}}{\partial u^{2}} s(h(x, u), u)\right|_{u=0}
\end{aligned}
$$

Proof. The proof follows the one of Theorem 2.6 in Byrnes and Lin [3].

(Necessity): If the system (1)-(2) is $Q S S$-lossless with supply function $s(y, u)$, and a storage function $V$, then

$$
\begin{aligned}
& V(f(x, u))-V(x)=s(h(x, u), u), \\
& \forall(x, u) \in \mathbb{X} \times \mathbf{u}
\end{aligned}
$$

Condition (9) is obtained substituting $u=0$ in (12). Conditions (10) and (11) follow from the first-order derivative and the second-order derivative of (12) with respect to $u$ and taking $u=0$.

(Sufficiency): Since $V$ is a $\mathscr{C}^{2}$ function and $V(f(x, u)$ ) is quadratic in $u$, the second-order Taylor's expansion formula of $V(f(x, u))$ at $u=0$ can be considered, and 


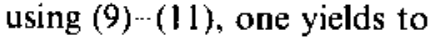

$$
\begin{aligned}
V(f(x, u))= & V(x)+s(h(x, 0), 0) \\
& +\left.\frac{\partial}{\partial u} s(h(x, u), u)\right|_{u=0} u \\
& +\left.\frac{1}{2} u^{\mathrm{T}} \frac{\partial^{2}}{\partial u^{2}} s(h(x, u), u)\right|_{u=0} u
\end{aligned}
$$

By claiming that $s(y, u)$ is quadratic in $u$, the secondorder Taylor expansion at $u=0$ of $s(h(x, u), u)$ can be used in (13) and equality (12) is obtained.

Remark 2.12. Conditions (5)-(6), (7) (8), and (9)-(11) are valid for multiple-input multipleoutput systems of the form (1)-(2) with $\mathscr{U}, \mathscr{Y}$ open subsets of $n^{m}$.

Necessary and sufficient conditions existing in the literature for lossless multi-input multi-output discrete-time systems of the form $x(k+1)=f(x(k))+$ $g(x(k)) u(k), \quad y(k)=h(x(k))+J(x(k)) u(k)$ are strictly contained in Theorem 2.11. For example, conditions appearing in Byrnes and Lin [3] are obtained taking $s(y, u)=y^{\mathrm{T}} u$. In order to obtain losslessness conditions presented in Sengör [27], the left-hand side of equality (9) would be $V(f(x)-x)$ and in (10) and (11), $z=f(x)-x$ should be considered with $V=B^{\mathrm{T}}(x)+$ $x^{\mathrm{T}} C(x) x$, where $B$ and $C$ are matrices of appropriate dimensions, and $s(y, u)=y^{\top} Q y+2 y^{\top} S u+u^{\top} R u$, where $Q, R, S$ are constant matrices of appropriate dimensions, and $Q, R$ are symmetric.

\subsection{Feedback Dissipativity and Feedback Passivity}

Let $\alpha: \mathscr{W} \times \mathscr{U} \rightarrow \mathscr{U}$ be a $\mathscr{C}^{\prime}$ function. A nonlinear static state feedback control law is denoted by the expression $u=\alpha(x, v)$. The system $x(k+1)=f(x(k)$, $\alpha(x(k), v(k)))$ is referred by the feedback transformed system, which may be also denoted by $x(k+1)=$ $\bar{f}(x(k), v(k))$. In addition, $\bar{h}(x, v)$ denotes the function $h(x, \alpha(x, v))$.

Definition 2.13. A feedback control law $u=\alpha(x, v)$ is locally regular if for all $(x, v) \in \mathscr{x} \times \mathscr{U}$, it follows that $\partial \alpha / \partial v \neq 0$

Definition 2.14. Consider the system (1)-(2) and two scalar functions $V(x)$ and $s(y, v)$ as a storage function and a supply function, respectively. The system is said to be feedback dissipative (resp., feedback stricly dissipative) with the functions $V$ and $s$ if there exists a regular static state feedback control law of the form $u=\alpha(x, v)$, with $v$ as the new input, such that the feedback transformed system is $(V, s)$-dissipative (resp., strictly $(V, s)$-dissipative).

The following theorem states sufficient conditions under which feedback dissipativity is possible.
Theorem 2.15. Consider the system (1)-(2) and two scalar functions $V(x)$ and $s(y, v)$ as a storage function and a supply function, respectively. Let $\phi(x, u)$ be a given dissipation rate function. Let $\left(x_{0}, u_{0}, v_{0}\right) \in$ $\mathscr{A}=\mathscr{R} \times \mathscr{U} \times \mathscr{H}$, with $\mathscr{A}$ an open set. Suppose that the following two conditions are satisfied:

1. $\exists\left(x_{0}, u_{0}, v_{0}\right)$ such that equality (4) holds for $s(y, v)$, i.e.,

$$
\begin{aligned}
& V\left(f\left(x_{0}, u_{0}\right)\right)-V\left(x_{0}\right) \\
& \quad=s\left(h\left(x_{0}, u_{0}\right), v_{0}\right)-\phi\left(x_{0}, u_{0}\right)
\end{aligned}
$$

$$
\begin{aligned}
& \text { 2. } \quad\left\{\frac{\partial}{\partial u}[V(f(x, u))-s(h(x, u), v) .\right. \\
& +\phi(x, u)]\}_{\left(x_{0}, u_{0}, v_{0}\right) \neq 0 .}
\end{aligned}
$$

Then, there exists a unique static state feedback control law of the form $u=\alpha(x, v)$ defined in a neighbourhood of $\left(x_{0}, v_{0}\right)$ and valued in a neigbourhood of $u_{0}$ such that the feedback transformed system $x(k+1)=\bar{f}(x(k), v(k)), y(k)=\bar{h}(x(k), v(k))$ is $(V, s)$ dissipative.

Proof. Let $\mathscr{X} \times \mathscr{M} \times \mathscr{U}$ an open set. Consider the following $\mathscr{C}^{1}$ function $F: \mathscr{W} \times \mathscr{U} \times \mathscr{U} \rightarrow \mathscr{T}$ defined by

$$
\begin{aligned}
F(x, v, u)= & V(f(x, u))-V(x) \\
& -[s(h(x, u), v)-\phi(x, u)]
\end{aligned}
$$

From condition (14), we have that $F\left(x_{0}, v_{0}, u_{0}\right)=0$. Condition (15) states that $\partial F / \partial u$ is non-singular at $\left(x_{0}, v_{0}, u_{0}\right)$. Then, by the implicit function theorem there exist open neighbourhoods $\bar{x} \subset \mathscr{X}$ of $x_{0}, \overline{\mathscr{U}}_{1} \subset \mathscr{U}$ of $v_{0}$ and $\overline{\mathscr{U}}_{2} \subset \mathscr{U}$ of $u_{0}$ and a unique map $G: \overline{\mathscr{Q}} \times \overline{\bar{u}}_{1} \rightarrow \overline{\mathscr{U}}_{2}$ such that

$$
F(x, v, G(x, v))=0, \quad \forall(x, v) \in \overline{\mathscr{T}} \times \overline{\mathscr{H}}_{1} .
$$

Thus, the implicit function theorem provides sufficient conditions which guarantee the existence of a local feedback control law $u=G(x, v)$ for the nonlinear equation (4) to be satisfied with $s(y, v)$; in other words, the existence of a control which renders system $(1)-(2)(V, s)$-dissipative with $s(y, v)$, and $v$ as the new input is guaranteed.

Definition 2.16. A system of the form (1)-(2) is said to be feedback passive if it is feedback dissipative with $s(y, v)=y v$.

Remark 2.17. Note that from Theorem 2.15 conditions for feedback dissipativity or passivation are the same as those for rendering a system $(V, s)$-lossless via 
static state feedback, considering $\phi(x, u)=0$, $\forall(x, u) \in: \mathbb{R} \times \mathscr{W}$.

\subsection{Dissipativity, Passivity and Stability}

All the results relating stability are given for a class of dissipative systems with supply function $s$ satisfying the ZIO property (3).

A nonlinear regular static state feedback control law of the form $u=\alpha(x, v)$, which achieves either $(V, s)$-dissipativity or strict $(V, s)$-dissipativity by means of static state feedback, induces an implicit damping injection which makes the system locally stable (resp., locally asymptotically stable if strict $(V, s)$-dissipativity is achieved) for certain particular values of the transformed control input.

Theorem 2.18. Consider the system (1) $\sim(2)$, and two scalar functions $V(x)$ and $s(y, v)$ as a storage function and a supply function satisfying the ZIO property, respectively. Suppose $\bar{x}$ an isolated fixed point for $f(x, \bar{u})$, with $\bar{u}$ a constant. Let $\phi(x, u)$ be a dissipation rate (resp., strict dissipation rate) function. Suppose there exists a feedback control law, $u=\alpha(x, v)$, defined in an open neighbourhood $\mathscr{W}=\tilde{X} \times \tilde{\mathscr{U}}$ with $\tilde{X} \subset \mathscr{X}, \tilde{\mathscr{U}} \subset \mathscr{\mathscr { H }}$ which achieves $(V, s)$-dissipativity (resp., strict $(V, s)$-dissipativity) of the feedback transformed system. Consider $x=\bar{x}$ the unique $x$ for which $V(x)=0$ and $\phi(x, \alpha(x, 0))=0$. Let $\mathscr{W}$ invariant with respect to $x(k+1)=f(x(k), \alpha(x(k), 0))$ and $(\bar{x}, \tilde{u}) \in \mathscr{W}$. Then, for all $x \in \overline{\mathscr{X}}$, the control law $u=\alpha(x, 0)$ locally stabilizes (resp., locally asymptotically stabilizes) the system to $\bar{x}$.

Proof. Since $u=\alpha(x, v)$ achieves strict $(V, s)$ dissipativity in an open neighbourhood $\mathscr{W}^{r}=\tilde{\mathscr{X}} \times \tilde{\mathscr{U}} \subset$ $\mathscr{X} \times \mathscr{U}$ (the argument is the same for $(V, s)$ dissipativity), relation (4) can be considered with $u=\alpha(x, v)$ and $s(y, v)$, then

$$
\begin{aligned}
& V(f(x, \alpha(x, v)))-V(x) \\
& \quad=s(h(x, \alpha(x, v)), v)-\phi(x, \alpha(x, v)), \forall(x, v) \in \mathscr{W}
\end{aligned}
$$

In particular, for $v=0$ and considering (3), (17) yields to,

$$
\begin{aligned}
& V(f(x, \alpha(x, 0)))-V(x) \\
& \quad=s(h(x, \alpha(x, 0)), 0)-\phi(x, \alpha(x, 0)) \\
& \quad=-\phi(x, \alpha(x, 0))<0, \quad \forall x \in \dot{x}
\end{aligned}
$$

Taking into account (18) and the fact that $x=\bar{x}$ is the unique $x$ for which $V(\bar{x})=0$ and $\phi(\bar{x}, \alpha(\bar{x}, 0))=0$, the result of the theorem follows from fundamental Lyapunov's Stability Theorem 2.5 .

Proposition 2.19. Consider the system (1)-(2) and two scalar functions $V(x)$ and $s(y, v)$ as a storage function and a supply function satisfying the ZIO property. respectively. Suppose $\bar{x}$ an isolated fixed point for $f(x, \bar{u})$. Let $\phi(x, u)$ be a dissipation rate (resp., strict dissipation rate) function. Let $V(\bar{x})=0$ and $\phi(\bar{x}, \bar{u})=0$. The system (1)-(2) is locally stabilizable (resp., asymptotically stabilizable) to $\bar{x}$ by the control $u=\alpha(x, 0)$ with $\alpha(x, v)$ a control law which renders the system $(V, s)$-dissipative (resp., strictly $(V, s)$ dissipative) if there exist $\left(x_{0}, u_{0}\right) \in X \times \mathscr{U}$ and $\mathscr{W}$ a neighbourhood of $\left(x_{0}, u_{0}\right)$ containing $(\bar{x}, \bar{u})$ for which the following relations are valid

1. $V\left(f\left(x_{0}, u_{0}\right)\right)-V\left(x_{0}\right)=-\phi\left(x_{0}, u_{0}\right)$,

2. $\left.\frac{\partial V(z)}{\partial z}\right|_{z=f(x, u)} \frac{\partial}{\partial u} f(x, u)+\frac{\partial}{\partial u} \phi(x, u) \neq 0$,

$\forall(x, u) \in \mathscr{W}$,

3. $\mathscr{W}$ invariant with respect to $x(k+1)=$ $f(x(k), \alpha(x(k), 0))$.

Proof. The result follows from Theorem 2.15 with $v=0$ and Theorem 2.18.

\section{The Energy Shaping Plus Damping Injection Method}

In this section, the passivity-based stabilization methodology of the Energy Shaping and Damping Injection (ESDI) is extended to the nonlinear discretetime case. The ESDI method consists in modifying the closed-loop system stored energy and in adding the required dissipation. In the literature, the ESDI idea has been applied in two different ways: the main difference between them is related to the way the energy shaping is performed. The approach of Ortega et al. [23] shapes the stored energy of the system for the desired equilibrium to be the minimum of the new energy function for the closed-loop system. On the other hand, in the classic passivitybased control approach Ortega and Spong [21], the definition of the controller is derived from a copy of the system with additional damping; here, the energy shaping is represented by the energy associated to an error dynamics, the definition of which is based on the proposal of an auxiliary dynamics, see e.g. $[30,31]$.

We will adapt the ESDI approach proposed in SiraRamirez and Navarro-López [31] to the discrete-time 
a detectability-type condition. Our proposal is more practical oriented.

\section{Example: a Discretized Model for the Buck Converter}

In this section, the ESDI control scheme is applied to a discrete-time model for the DC-to-DC buck converter. Although the model is linear, it is appropriate to illustrate the proposed dissipativity-based control methodology. It is an example for which the energy concepts introduced have a physical interpretation.

The following system is a discrete-time model of a normalized averaged DC-to-DC buck converter [9], a well known physical system employed in power electronics,

$$
\begin{aligned}
\left(\begin{array}{c}
x_{1}(k+1) \\
x_{2}(k+1)
\end{array}\right)= & \left(\begin{array}{cc}
a & -b \\
b & c
\end{array}\right)\left(\begin{array}{c}
x_{1}(k) \\
x_{2}(k)
\end{array}\right) \\
& +\hat{u}(k)\left(\begin{array}{c}
\gamma(-a+1)+b \\
-\gamma b-c+1
\end{array}\right), \\
& \hat{u} \in[0,1]
\end{aligned}
$$

where $x_{1}$ is a current, $x_{2}$ a voltage, $\gamma$ the normalized load and $a, b, c$ constants related to physical parameters.

For the normalized variables the stored system energy takes the following form:

$$
E_{\mathrm{S}}=\frac{1}{2}\left(x_{1}^{2}+x_{2}^{2}\right)
$$

We aim to stabilize the output voltage $x_{2}$ to a constant value $\bar{x}_{2} \in\{0,1]$. The energy associated to the system (28) is considered as the storage function $V$, and $s(y, v)=y v=x_{2} v$ as the supply function. Let $u$, $v \in[0,1], x_{1} \in[0, \gamma \rho], x_{2} \in[0, \rho]$, with $\rho>1$. First of all, a function $\phi$ must be proposed. This function will be chosen in order to collect the positive terms appearing in $V(x(k+1))$. Consequently, a possibility for $\phi$ is the following one,

$$
\begin{aligned}
\phi(x, \hat{u})= & \mu\left\{x_{1}^{2}\left(a^{2}+b^{2}\right)+x_{2}^{2}\left(b^{2}+c^{2}\right)\right. \\
& +\hat{u}^{2}\left[\gamma^{2}(-a+1)^{2}+b^{2}\left(1+\gamma^{2}\right)\right. \\
& \left.\left.+(-c+1)^{2}\right]\right\},
\end{aligned}
$$

where $\mu$ is a constant such that $\mu \in(0,0.137817)$. The constant $\mu$ represents the damping injection to the system; the smaller $\mu$ is, the slower the convergence to the fixed point is. The stabilization of the system strongly depends on the form of $\phi$. The ESDI control scheme is applied and four main steps can be distinguished it it, namely:

Step I Computation of the control $\alpha(e, 0)$ from

$$
V(f(e, \alpha(e, 0)))-V(e)=-\phi(e, \alpha(e, 0)),
$$

which results in a second-order equation of the form $a_{\alpha} \alpha^{2}+b_{\alpha} \alpha+c_{\alpha}=0$, where

$$
\begin{aligned}
a_{\alpha}= & \left(\frac{1}{2}+\mu\right)\left[\gamma^{2}(-a+1)^{2}+b^{2}\left(\gamma^{2}+1\right)\right. \\
& \left.+(-c+1)^{2}\right]+\gamma\left(b c^{2}-a b\right), \\
h_{\alpha}= & {[\gamma(-a+1)+b]\left(a e_{1}-b e_{2}\right) } \\
& +[-\gamma b-c+1]\left(b e_{1}+c e_{2}\right), \\
c_{\alpha r}= & \left(\frac{1}{2}+\mu\right)\left[e_{1}^{2}\left(a^{2}+b^{2}\right)+e_{2}^{2}\left(b^{2}+c^{2}\right)\right] \\
& +(b c-a b) e_{1} e_{2}-\frac{1}{2}\left(c_{2}^{2}+e_{1}^{2}\right),
\end{aligned}
$$

with $e=\left(e_{1}, e_{2}\right)^{\mathrm{T}}=\left(x_{1}-\xi_{1}, x_{2}-\xi_{2}\right)^{\mathrm{T}}, \quad \xi=\left(\xi_{1}, \xi_{2}\right)^{\mathrm{T}}$. Passivation conditions (14) and (15), are transformed for stabilization purposes in conditions (19)-(20) which are met for this example if,

$$
a_{\alpha} \alpha^{2}+b_{\alpha} \alpha+c_{\alpha}=0, \quad 2 a_{\alpha} \alpha+b_{\alpha} \neq 0,
$$

for some $(e, \alpha)$. If conditions (31) are satisfied, $\alpha$ exists. This $\alpha$ can be obtained from the explicit solution of (30), then, it is necessary to assure that $b_{\alpha}^{2}-4 a_{\alpha} c_{\alpha} \geq 0$, which will be achieved by means of the value of $\mu \in(0,0.137817)$.

Step 2 Computation of the stabilizing control $\hat{u}^{*}$ from the proposed auxiliary dynamics,

$$
\begin{aligned}
\xi_{1}(k+1)= & a x_{1}(k)-b x_{2}(k)+[\gamma(-a+1)+b] \hat{u}(k) \\
& -f_{1}(e(k), \alpha(e(k), 0)), \\
\xi_{2}(k+1)= & b x_{1}(k)+c x_{2}(k)+(-\gamma b-c+1) \hat{u}(k) \\
& -f_{2}(e(k), \alpha(e(k), 0)),
\end{aligned}
$$

with $f=\left(f_{1}, f_{2}\right)^{\mathrm{T}}$. We want to stabilize $\xi_{2}$ to a constant value $\bar{x}_{2} \in(0,1]$ then $\xi_{2}(k)=\bar{\xi}_{2}=\bar{x}_{2}, \forall k$, and $\hat{u}^{*}$ is obtained from (33),

$$
\hat{u}^{*}=\frac{\bar{x}_{2}-\left(b x_{1}+c x_{2}\right)+f_{2}(e, \alpha(e, 0))}{(-\gamma b-c+1)} .
$$

Step 3 Computation of the auxiliary dynamics (32) using $\hat{u}^{*}$.

Step 4 Computation of the system state. Control $\hat{u}^{*}$ is applied to (27).

The previous control design has been applied to (27) with the following parameters obtained from a real physical system: $a=0.9406416964, b=0.3254699438$, $c=0.8255706942, \gamma=0.3535533906$ and a sampling period of $T=0.3535533906$. The fixed point of system (27) is $\bar{x}_{1}=\gamma \hat{\bar{u}}, \bar{x}_{2}=\hat{\bar{u}}$. Considering $\bar{x}_{2}=0.2$, with $x_{0}=(0.01,0.05)^{T}, \xi_{10}=0.01$ as initial conditions for $x$ 

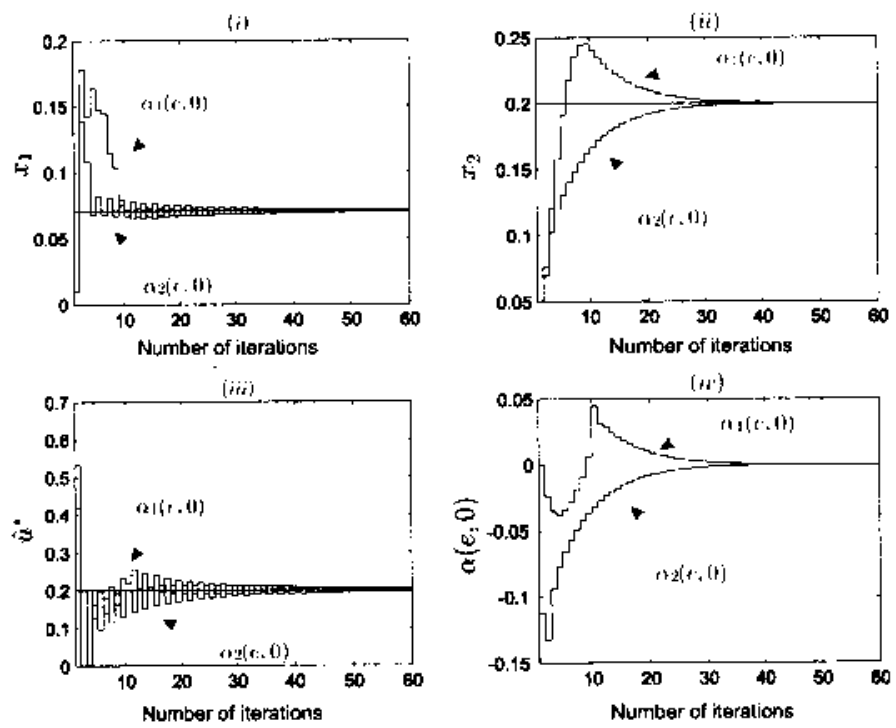

Fig. 2. Normalized current $x_{1}$, normalized voltage $x_{2}$, stabilizing control $\hat{u}^{*}$ and passifying controls for $\alpha_{1}(e, 0)$ and $\alpha_{2}(e, 0)$.

and $\xi_{1}$, respectively, and $\mu=0.135$, the system response presented in Fig. 2 is obtained. The system converges to its fixed point. There are two possible solutions for $\alpha(e, 0)$ :

$$
\begin{aligned}
& \alpha_{1}(e, 0)=\frac{-b_{\alpha}+\sqrt{b_{\alpha}^{2}-4 a_{\alpha} c_{\alpha}}}{2 a_{\alpha}}, \\
& \alpha_{2}(e, 0)=\frac{-b_{\alpha}-\sqrt{b_{\alpha}^{2}-4 a_{\alpha} c_{\alpha}}}{2 a_{\alpha}} ;
\end{aligned}
$$

the responses obtained from each of them are slightly different. The difference between considering $\alpha_{1}(e, 0)$ or $\alpha_{2}(e, 0)$ can be seen in the type of transient response for the obtained output voltage $x_{2}$. Using the latter, a first-order type system response for the voltage without overshooting is obtained. Both responses have the same settling time. The states $x_{1}, x_{2}$, the stabilizing control $\hat{u}^{*}$, and the passifying controls $\alpha_{1}(e, 0)$ and $\alpha_{2}(e, 0)$ are depicted in Fig. 2.

\section{Conclusions}

In this article, the notions of dissipativity, passivity, feedback dissipativity and passivation for general single-input single-output nonlinear discrete-time systems have been analyzed. Necessary conditions for the characterization of a class of dissipative nonlinear discrete-time systems have been given. Necessary and sufficient conditions fulfilled by a class of lossless systems regarded as $Q S S$-lossless systems have been also derived.

Sufficient conditions under which a class of nonaffine discrete-time control systems are feedback dissipative have been given. The feedback dissipativity and passivation problems have been solved in a nongeneral manner since they are based on the establishment of the input $u$ which satisfies the dissipativity fundamental inequality; it is, therefore, necessary to associate a priori functions $V$ and $\phi$ to the system, i.e., a storage function and a dissipation rate function with respect to which the feedback transformed system will be $(V, s)$-dissipative. At any rate, it can be considered as an application-oriented feedback dissipativity method, since, when dealing with physical systems, we are interested in defining our storage function as the energy of the system, and proposing a desired dissipation. This fact is shown in the buck example. The main problem of this procedure is that finding an explicit solution of $u$ for all $k$ can be difficult or impossible in some systems; in these cases, an iterative feedback dissipativity algorithm can be proposed [20].

As for the stabilization purpose, stability properties of dissipative and feedback dissipative systems have been analyzed and the ESDI controller design methodology has been extended to general nonlinear systems in the discrete-time domain. This control scheme has been illustrated by means of the stabilization of the voltage in a proposed discrete-time model for the buck converter.

\section{Acknowledgements}

The authors gratefully acknowledge the efforts of the anonymous reviewers, who suggested valuable and helpful comments. 


\section{References}

1. Byrnes CI, Isidori A, Willems JC. Passivity, feedback equivalence, and the global stabilization of minimum phase nonlinear systems. IEEE Trans Automa Control 1991; 36: 1228-1240

2. Byrnes CI, Lin W. Discrete-time lossless systems, feedback equivalence, and passivity. Proceedings of the 32nd Conference on Decision and Control, San Antonio, Texas. 1993, pp 1775-1781

3. Byrnes CI, Lin W. Losslessness, feedback equivalence, and the global stabilization of discrete-time nonlinear systems. IEEE Trans Autom. Control 1994; 39(1): $83-98$

4. Fradkov AL, Pogromsky AY. Introduction to Control, Oscillations and Chaos. World Scientific Publishing Co. 1998.

5. Göknar IC, Sengör NS. Discrete-time version of Kalman-Yakubovich Popov lemma for non-linear systems. Proceedings of the 5th International Conference on Electronics, vol 1 Circuits and Systems, Lisboa, Portugal 1998, pp 79 -82

6. Hill DJ, Moylan P. The stability of nonlinear dissipative systems. IEEE Trans Autom Control 1976; 21: $708-711$

7. Hill DJ, Moylan P. Dissipative dynamical systems: basic input-output and state properties. J Franklin Inst 1980; 309: 327-357

8. Hitz L, Anderson BDO. Discrete positive-real functions and their application to system stability. Proc of IEE 1969; 116: 153-155

9. Kassakian JG, Schlecht MF, Verghese GC. Principles of Power Electronics. Addison Wesley 1991

10. Khalil HK. Nonlinear systems. Prentice-Hall, 1996.

11. Kokotovic PV, Sussmann HJ. A positive real condition for global stabilization of nonlinear systems. Syst Control Lett 1989; 13: 125-133

12. LaSalle JP. The stability and control of discrete processes. Springer-Verlag, Berlin Heidelberg, New York 1986.

13. Lin W. Synthesis of discrete-time nonlinear systems. Ph.D. Thesis, Washington University 1993.

14. Lin W. Feedback stabilization of general nonlinear control systems: a passive system approach. Syst Control Lett 1995; 25: 4l-52

15. Lin W, Byrnes CI. Passivity and absolute stabilization of a class of discrete-time nonlinear systems. Automatica 1995; 31(2): 263-267

16. Lin W, Byrnes CI. Zero-state observability and stability of discrete-time nonlinear systems. Automatica 1995; 31(2): 269-274

17. Lin W. Global asymptotic stabilization of general nonlinear systems with stable free dynamics via passivity and bounded feedback. Automatica 1996; 32(6): $915-924$

18. Moylan P. Implications of passivity in a class of nonlinear systems. IEEE Trans Autom Control 1974; AC-19(4): $373-381$
19. Monaco S, Normand-Cyrot D. On the conditions of passivity and losslessness in discrete time. Proceedings of the European Control Conference 1997.

20. Navarro-López EM, Fossas-Colet E, Cortés D. Passification of nonlinear discrete-time systems using the speed-gradient atgorithm. Proceedings of the National Congress of Mexico Cont. Aut. Assoc., San Luis Potosí. 2001, pp 14]-146

21. Ortega R, Spong M. Adaptive motion control of rigid robots: a tutorial. Automatica 1989; 25(6): 877-888

22. Ortega R, Loria A, Nicklasson PJ, Sira-Ramirez H. Passivity-based control of Euler-Lagrange systems. Mechanical, electrical and electromechanical applications. Springer-Verlag, London 1998.

23. Ortega $R$, van der Schaft AJ, Maschke B, Escobar G. Energy-shaping of port-controlled Hamiltonian systems by interconnection. Proceedings of the 38th Conference on Decision and Control, Phoenix 1999, pp $1646-1651$

24. Popov VM. Hyperstability of control systems. SpringerVerlag, Berlin Heidelberg. New York 1973.

25. Santosuosso GL. Passivity of nonlinear systems with input-output feedthrough. Automatica 1997; 33(4): 693-697

26. van der Schaft AJ. $L_{2}$-gain and passivity techniques in nonlinear control. Lecture notes in control and information sciences, vol 218, Springer-Verlag, 1996.

27. Sengör NS. Energy related concepts in nonlinear systems. Ph.D. Thesis, Istanbul Technical University, Institute of Science and Technology, Instanbul 1995.

28. Sepulchre R, Janković M, Kokotović P. Constructive nonlinear control. Springer-Verlag, Berlin Hejdelberg, New York 1997.

29. Shiriaev AS. The notion of $V$-detectability and stabilization of invariant sets of nonlinear systems. Syst and Control Lett 2000; 39: 327-338

30. Sira-Ramírez $\mathbf{H}$. A general canonical form for feedback passivity. International Journal of Control, Special Issue: Recent Adv Control Nonlinear Syst 1998; 71(5): $891-905$

31. Sira-Ramirez H, Navarro-López EM. On the passivity of general nonlinear systems. Proceedings of the 14 th Symposium on Mathematical Theory of Networks and Systems, Perpignan, France. June 2000.

32. Willems JC. Dissipative dynamical systems. Part I: general theory. Part II: linear systems with quadratic supply rates. Arch Rational Mech Anal 1972; 45(5): $321-393$

33. $\mathrm{Wu} \mathrm{M}$, Desoer $\mathrm{CA}$. Input-output properties of multiple-input, multiple-output discrete systems: Part II. J Franklin Inst 1970; 290(2): 85-101

34. Zames $G$. On the input-output stability of timevarying nonlinear feedback systems. Part I: conditions derived using concepts of loop gain, conicity, and positivity. IEEE Trans Autom Control 1966; $A C-11(2): 465-476$ 